\title{
A modified formula for calculating low-density lipoprotein cholesterol values
}

\author{
Yunqin Chen ${ }^{+1}$, Xiaojin Zhang ${ }^{+2}$, Baishen Pan'1, Xuejuan Jin'1, Haili Yao ${ }^{3}$, Bin Chen¹, Yunzeng Zou'1, Junbo Ge ${ }^{* 1}$ and \\ Haozhu Chen*1
}

\begin{abstract}
Background: The Friedewald formula (FF) is useful for calculating serum low-density lipoprotein cholesterol (LDL-C) values, but has a remarkable deviation and limitation especially in hypertriglyceridemia. We modify the formula which is now more suitable for $\mathrm{LDL}-\mathrm{C}$ calculation.

Methods: 2180 cases were classified into three groups according to their TG concentrations ( $\mathrm{A}:<200 \mathrm{mg} / \mathrm{dl}, n=1220$; B: 200-400 mg/dl, $n=480 ;$ C: 400-1000 mg/dl, $n=480$ ). The concentrations of LDL-C were measured or estimated by 1) a direct measurement (DM); 2) the FF; and 3) our modified Friedewald formula (MFF): LDL-C (mg/dl) = Non-HDL-C $\times$ $90 \%-\mathrm{TG} \times 10 \%$.

Results: Linear regression showed a significant correlation $(P<0.001)$ between the measured and calculated LDL-C values. Bland-Altman plots indicated that the methods (DM/MFF) were in better agreement than those (DM/FF). The LDL-C/Non-HDL-C ratio in FF calculated values was significantly lower $(P<0.05)$ than that in MFF or DM values, while no significant difference between MFF and DM was found. In Group A and Group B, 4.26\% and $14.79 \%$ of the MFF calculated values had more than $20 \%$ deviation from those measured by DM. These percentages were significantly lower than those calculated by FF, where $7.30 \%$ and $25.63 \%$ were observed, respectively $(P<0.01$ and $P<0.001)$. The MFF calculated values were all positive even in Group C.

Conclusions: Compared with the FF calculation, serum LDL-C values estimated by our modified formula are closer to those measured by a direct assay. The modification significantly diminishes the interference caused by

hypertriglyceridemia.
\end{abstract}

\section{Introduction}

Serum low-density lipoprotein cholesterol (LDL-C) is an independent risk factor for the development of coronary heart disease [1]. The National Cholesterol Education Program Adult Treatment Panel and other scientific societies have identified LDL-C concentrations as the primary criterion of diagnosis and treatment of patients with hyperlipidemia $[2,3]$.

Most clinical laboratories estimated LDL-C concentrations in serum by the Friedewald formula (FF) from the concentrations of total cholesterol (TC), triglyceride (TG), and high-density lipoprotein cholesterol (HDL-C) [4-6]. The traditional FF is: LDL-C $(\mathrm{mg} / \mathrm{dl})=$ Non-HDLC - TG/5. TG is mainly from chylomicrons and VLDL.

*Correspondence: ge.junbo@zs-hospital.sh.cn, chen.haozhu@zs-hospital.sh.cn Zhongshan Hospital, Fudan University, Shanghai, China, 200032

† Contributed equally

Full list of author information is available at the end of the article
Assuming Non-HDL-C has little or no change, if TG levels are too high, the LDL-C values would be underestimated. This could occur in the postprandial condition or patient with normal Non-HDL-C but high TG levels. The FF estimated value is not valid in specimens with TG more than $400 \mathrm{mg} / \mathrm{dl}[7,8]$. Indeed, it has been recommended that the FF should be used with precaution in several pathologic states (diabetes, hepatopathy, nephropathy), even if the TG concentrations are between 200 $\mathrm{mg} / \mathrm{dl}$ and $400 \mathrm{mg} / \mathrm{dl}[9,10]$. In previous reports, the formula was modified to overcome the limitation [11-15]. However, these modifications were either complicated or lacking rationales. From the FF, we can see LDL-C is determined by the correlated parameters of none highdensity lipoprotein cholesterol (Non-HDL-C) and TG. We may reach to a suitable point through adjusting both parameters. We proposed a modified Friedewald formula 
(MFF). Based on the formula, we calculated LDL-C values which were compare with the FF and a direct homogeneous assay.

\section{Materials and methods}

Blood samples were obtained from 2180 adult outpatients, ages $>18$ years, at the department of clinical laboratory of Zhongshan Hospital. Blood was collected in tubes without anticoagulant from subjects after an overnight fast. The samples were allowed to clot at room temperature, and serum was obtained by centrifugation at $3000 \mathrm{rpm}$ for 15 minutes. All blood lipid analyses were performed within 1 day. All subjects were classified into three groups according to the TG concentrations (A: < $200 \mathrm{mg} / \mathrm{dl}, n=1220$; B: $200-400 \mathrm{mg} / \mathrm{dl}, n=480$; C: $400-$ $1000 \mathrm{mg} / \mathrm{dl}, n=480$ ). The Non-HDL-C concentrations in all samples were less than $300 \mathrm{mg} / \mathrm{dl}$. To convert values for TG and cholesterol to millimoles per liter, we multiply the values with 0.0113 and 0.0259 , respectively.

The Non-HDL-C value was estimated by the formula as follows [16]:

$$
\text { Non }-\mathrm{HDL}-\mathrm{C}=\mathrm{TC}-\mathrm{HDL}-\mathrm{C}
$$

Lipid measurements were performed on a Hitachi 911 automatic analyzer. The LDL-C assay was performed according to Roche manufacture's specifications. At the same time, the LDL-C values were also calculated by the FF and MFF. TC and TG concentrations were determined enzymatically using CHOD-PAP and lipase/GPO/PAP methods, respectively. The HDL-C concentration was measured by phosphotungstic acid and $\mathrm{MgCl}_{2}$ precipitation approach. The reagents were obtained from Roche Diagnostics. The procedures and efficiency of lipid assays had been demonstrated previously [17]. The total error used in precision assessment was 3.95\%-7.85\% for the Roche method, as recommended by the National Cholesterol Education Program.

The FF was transformed as follows:

$$
\mathrm{LDL}-\mathrm{C}(\mathrm{mg} / \mathrm{dl})=\mathrm{Non}-\mathrm{HDL}-\mathrm{C}-\mathrm{TG} \times 20 \%
$$

Multivariate linear regression analysis was used to investigate the relationship between LDL-C (expected value), TG and Non-HDL-C (explanatory variables) concentrations. Repeatability of the new formula was evaluated by Bland-Altman analysis [18]:. We compared the agreement between FF and our new formula, and calculated the mean and standard deviation of the differences (formula and lab value). The mean difference of both FF and new formula were close to zero. We concluded the MFF as follows:

$$
\mathrm{LDL}-\mathrm{C}(\mathrm{mg} / \mathrm{dl})=\mathrm{Non}-\mathrm{HDL}-\mathrm{C} \times 90 \%-\mathrm{TG} \times 10 \%
$$

Statistical analysis was performed using SPSS 11.5 for Windows (SPSS Inc., USA). Linear regression analyses were used to assess the correlations between the methods

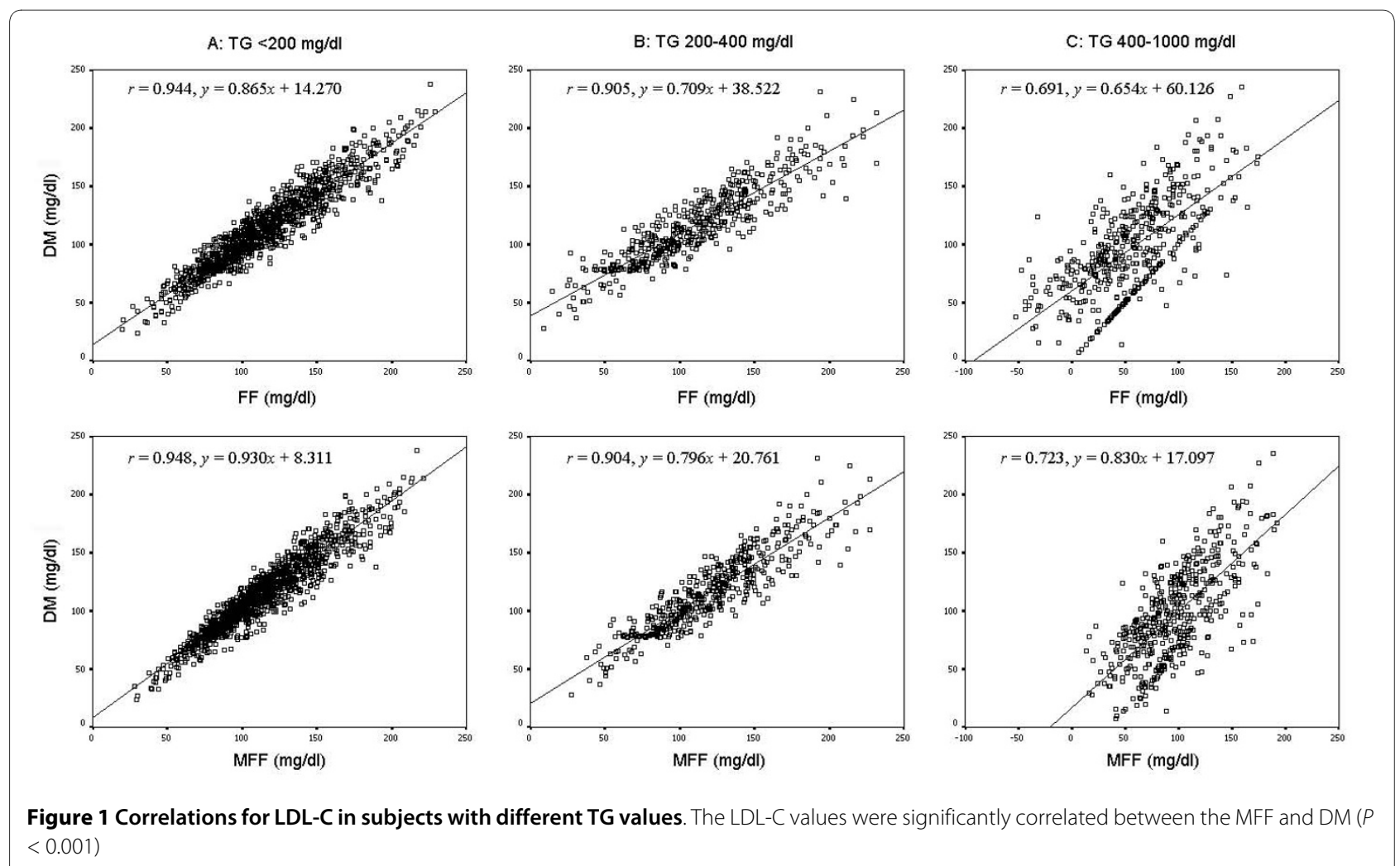




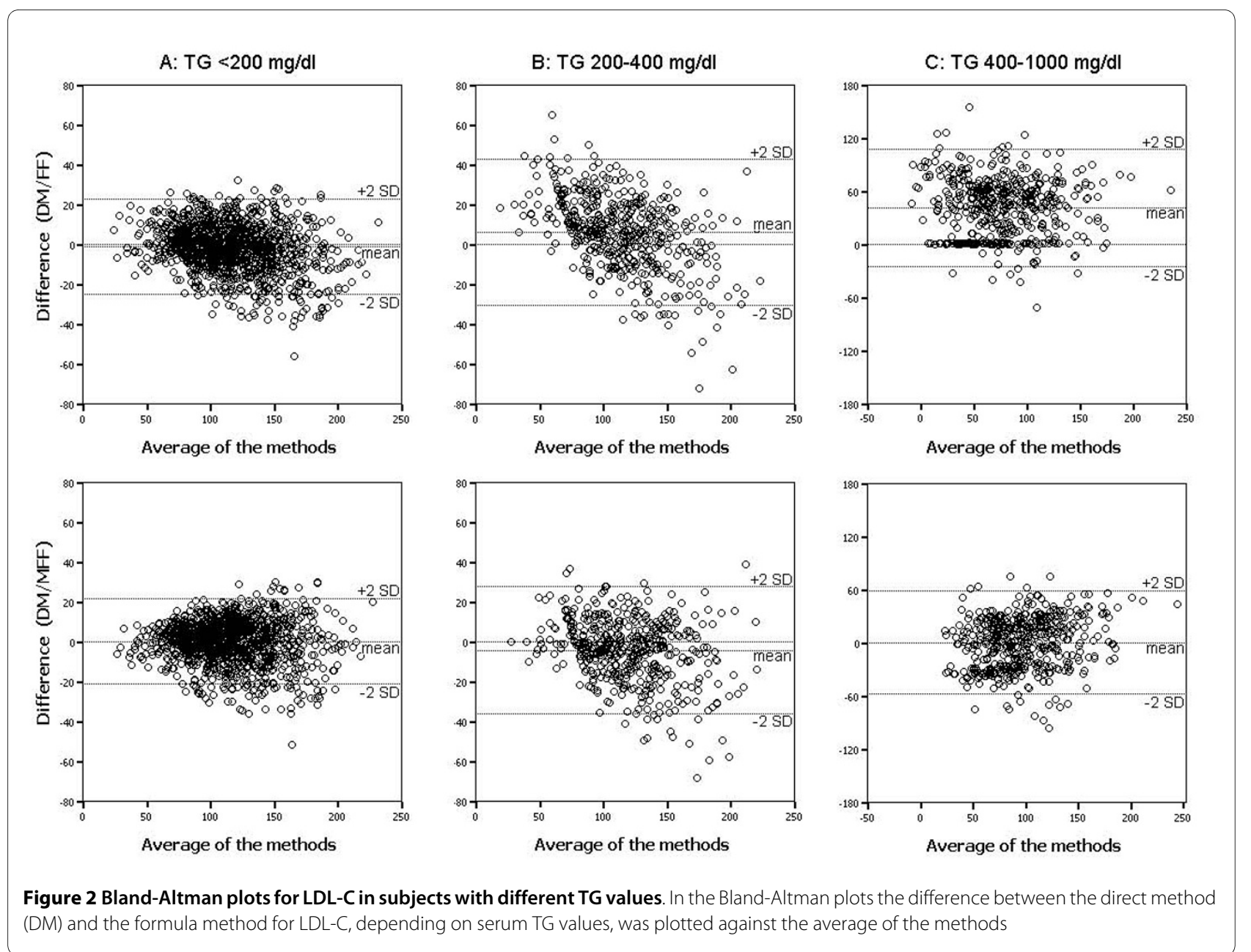

of formula calculation and direct measurement. To examine the degree of consistency between values obtained by the two methods, we used the graphical procedure outlined by Bland and Altman. Comparisons between groups were performed using the method of ANOVA. The test of Pearson chi-square was used to compare discrete variables. $P$ values less than 0.05 were considered significant.

\section{Results}

As shown in the Figure 1, the LDL-C values were significantly correlated between the MFF and DM $(P<0.001)$. In the group with $\mathrm{TG}>400 \mathrm{mg} / \mathrm{dl}$, our formula but not traditional FF fit well with the DM. As shown in the Figure 2, the difference between the $\mathrm{DM}$ and the formula methods for LDL-C, depending on the TG values, was plotted against the average of the methods. The BlandAltman plots indicated a good agreement between the methods of DM and MFF. As shown in the Figure 3, the ratios of LDL-C to Non-HDL-C values were significantly decreased in the subjects with hypertriglyceridemia. This ratio in FF calculated values was significantly lower $(P<$ $0.05)$ than that in MFF or DM values, even in Group B
(TG: 200-400 mg/dl), while no significant difference between the MFF and DM was found.

In order to evaluate MFF is better than FF, we also calculated LDL-C values using MFF and FF in Group A (TG $<200 \mathrm{mg} / \mathrm{dl}$ ) and Group B (TG: $200-400 \mathrm{mg} / \mathrm{dl}$ ) and then compared these values with that measured by DM. We found that $4.26 \%$ and $14.79 \%$ of the values calculated by the MFF had more than $20 \%$ deviation form that measured by DM. These percentages were significantly lower than those calculated by FF, where $7.30 \%$ and $25.63 \%$ were observed, respectively $\left(X^{2}=10.305, P<0.01\right.$ and $X^{2}$ $=17.468, P<0.001)$, suggesting MFF works better than FF in predicting LDL-C values at least in these populations. We compared LDL-C values measured by MFF or FF with those measured by DM. We found that all MFF calculated values were positive ones, while $10.42 \%$ of FF calculated values were negative. The results indicated our modified formula could provide a better estimate of LDL$\mathrm{C}$ values than $\mathrm{FF}$.

\section{Discussion}

The LDL-C value is estimated using the FF, which can be transformed as follows: LDL-C (mg/dl) = Non-HDL-C - 
TG $\times 20 \%$. In the formula, the concentration of very lowdensity lipoproteins (VLDL) is estimated as $20 \%$ of total TG concentrations [4]. However, the particles found in patients with hypertriglyceridemia are usually a heterogeneous mixture of chylomicron remnants, VLDL, and VLDL remnants [19]. It is well known that the ratio of TG to cholesterol (TG/cholesterol) varies a lot within these particles. When the TG concentration is more than 400 $\mathrm{mg} / \mathrm{dl}$, the cholesterol in TG-rich lipoproteins is overestimated by the FF method, resulting that the calculated LDL-C value even appears negative [20]. Therefore, this method has a limitation in clinical application.

In this study, the FF estimated value was calibrated using different coefficients to avoid LDL-C underestimation. We examined the correlation between traditional FF and our new formula, and found that both of FF and MFF fit well with the DM in the subjects with TG $<400 \mathrm{mg} / \mathrm{dl}$. While If the TG concentrations were higher than $400 \mathrm{mg} /$ $\mathrm{dl}$, our formula but not traditional FF fit well with the DM. However, because high coefficients do not necessarily mean that two methods agree. We assessed the degree of agreement between the two methods using the BlandAltman graphical technique. The Bland-Altman graphs are plots of the difference between the two methods against their mean. The degree of agreement is indicated by calculating the bias, estimated by the mean and SD of the differences. The figure 2 showed an obvious relationship between the differences and the mean. The BlandAltman plots suggest that the methods (DM/MFF) are in better agreement than those (DM/FF). Moreover, The MFF calculated values had a smaller deviation from the DM values and a regular relationship with the Non-HDL$C$ values, showing our new formula works better than classical FF.

In summary, a modified formula for LDL-C calculation was designed in this study: LDL-C $(\mathrm{mg} / \mathrm{dl})=$ Non-HDL-C

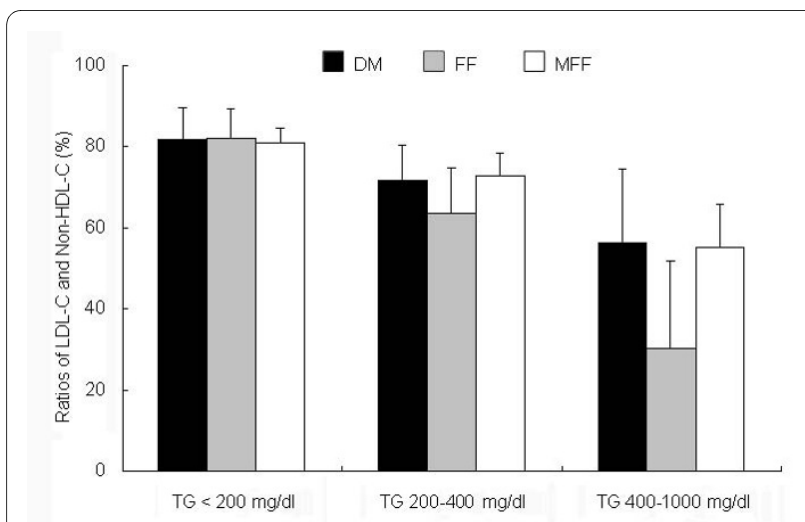

Figure 3 Ratios of LDL-C to Non-HDL-C values in subjects with different TG values. The $\mathrm{LDL}-\mathrm{C} / \mathrm{Non}-\mathrm{HDL}-\mathrm{C}$ ratio in FF calculated values was significantly lower in the groups with TG>200 mg/dl than that in MFF or DM values ( $P<0.05$ ), even in Group B (TG: $200-400$ mg/dl), while no significant difference between the MFF and DM was found $\times 90 \%-$ TG $\times 10 \%$. The MFF estimated LDL-C values have following characteristics: 1 ). They are closer to those measured by the DM than those estimated by the FF; 2 ). They have a stable LDL-C/Non-HDL-C ratio; and 3). The interference caused by hypertriglyceridemia might be significantly diminished. Therefore, the MFF has higher accuracy than FF. Although our findings need to be confirmed in additional studies, they hold a promise of broadening the usage of the FF in LDL-C measurement.

\section{Competing interests}

The authors declare that they have no competing interests.

\section{Authors' contributions}

YC and XZ carried out the data analysis, provided the hypothesis and drafted the manuscript. BP carried out clinical assays of blood lipids. XJ performed the statistical analysis. HY and BC collected and managed the blood samples. YZ, $J G$ and $H C$ conceived of the study, and participated in its design and coordination.

All authors read and approved the final manuscript.

\section{Acknowledgements}

This work was supported by grants from National Natural Science Foundation of China (No. 30500205, No. 30873351 and No. 30571602). We thank Jong Wu and Weiyun Wu (Department of Clinical Laboratory of Zhongshan Hospital) for their support.

\section{Author Details}

1Zhongshan Hospital, Fudan University, Shanghai, China, 200032, 2Obstetrics \& Gynecology Hospital, Fudan University, Shanghai, China, 200011 and

${ }^{3}$ Children's Hospital, Fudan University, Shanghai, China, 200032

Received: 12 April 2010 Accepted: 21 May 2010

Published: 21 May 2010

\section{References}

1. Cheng AY, Leiter LA: Implications of recent clinical trials for the National Cholesterol Education Program Adult Treatment Panel III guidelines. Curr Opin Cardiol 2006, 21:400-4.

2. National Cholesterol Education Program (NCEP) Expert Panel: Third Report of the National Cholesterol Education Program (NCEP) Expert Panel on Detection, Evaluation, and Treatment of High Blood Cholesterol in Adults (Adult Treatment Panel III) final report. Circulation 2002, 106:3143-421.

3. Keevil JG, Cullen MW, Gangnon R, McBride PE, Stein JH: Implications of cardiac risk and low-density lipoprotein cholesterol distributions in the United States for the diagnosis and treatment of dyslipidemia: data from National Health and Nutrition Examination Survey 1999 to 2002. Circulation 2007, 115:1363-70.

4. Friedewald WT, Levy RI, Fredrickson DS: Estimation of the concentration of low-density lipoprotein cholesterol in plasma, without use of the preparative ultracentrifuge. Clin Chem 1972, 18:499-502.

5. McNamara JR, Cohn JS, Wilson PW, Schaefer EJ: Calculated values for low-density lipoprotein cholesterol in the assessment of lipid abnormalities and coronary disease risk. Clin Chem 1990, 36:36-42.

6. Abudu N, Levinson SS: Calculated low-density lipoprotein cholesterol remains a viable and important test for screening and targeting therapy. Clin Chem Lab Med 2007, 45:1319-25.

7. Nauck M, Warnick GR, Rifai N: Methods for measurement of LDLcholesterol: a critical assessment of direct measurement by homogeneous assays versus calculation. Clin Chem 2002, 48:236-54

8. Lindsey CC, Graham MR, Johnston TP, Kiroff CG, Freshley A: A clinical comparison of calculated versus direct measurement of low-density lipoprotein cholesterol level. Pharmacotherapy 2004, 24:167-72.

9. Tighe DA, Ockene IS, Reed G, Nicolosi R: Calculated low density lipoprotein cholesterol levels frequently underestimate directly measured low density lipoprotein cholesterol determinations in patients with serum triglyceride levels $<$ or $=4.52 \mathrm{mmol} / \mathrm{l}$ : an analysis 
comparing the LipiDirect magnetic LDL assay with the Friedewald calculation. Clin Chim Acta 2006, 365:236-42

10. Rubiés-Prat J, Reverter JL, Sentí M, Pedro-Botet J, Salinas I, Lucas A, Nogués $X$, Sanmartí A: Calculated low-density lipoprotein cholesterol should not be used for management of lipoprotein abnormalities in patients with diabetes mellitus. Diabetes Care 1993, 16:1081-6.

11. Gazi I, Tsimihodimos V, Filippatos TD, Saougos VG, Bairaktari ET, Tselepis $A D$, Elisaf $M: L D L$ cholesterol estimation in patients with the metabolic syndrome. Lipids Health Dis 2006, 5:8.

12. Wägner AM, Zapico E, Bonet R, Pérez A, Ordóñez-Llanos J: The effect of VLDL particles on the accuracy of a direct LDL-cholesterol method in type 2 diabetic patients. Clin Biochem 2003, 36:177-83.

13. Planella T, Cortés M, Martínez-Brú C, González-Sastre F, Ordóñez-Llanos J: Calculation of LDL-cholesterol by using apolipoprotein B for classification of nonchylomicronemic dyslipemia. Clin Chem 1997, 43:808-15.

14. Hattori Y, Suzuki M, Tsushima M, Yoshida M, Tokunaga Y, Wang Y, Zhao D, Takeuchi M, Hara Y, Ryomoto Kl, Ikebuchi M, Kishioka H, Mannami T, Baba S, Harano Y: Development of approximate formula for LDL-chol, LDLapo $B$ and $L D L$-chol/LDL-apo $B$ as indices of hyperapobetalipoproteinemia and small dense LDL. Atherosclerosis 1998, 138:289-99.

15. Walldius G, Jungner I, Holme I, Aastveit AH, Kolar W, Steiner E: High apolipoprotein B, low apolipoprotein A-I, and improvement in the prediction of fatal myocardial infarction (AMORIS study): a prospective study. Lancet 2001, 358:2026-33.

16. Miller M, Ginsberg HN, Schaefer EJ: Relative atherogenicity and predictive value of non-high-density lipoprotein cholesterol for coronary heart disease. Am J Cardiol 2008, 101:1003-8.

17. Esteban-Salán M, Guimón-Bardesi A, de La Viuda-Unzueta JM, AzcarateAnia MN, Pascual-Usandizaga P, Amoroto-Del-Río E: Analytical and clinical evaluation of two homogeneous assays for LDL-cholesterol in hyperlipidemic patients. Clin Chem 2000, 46(8 Pt 1):1121-31.

18. Bland JM, Altman DG: Statistical methods for assessing agreement between two methods of clinical measurement. Lancet 1986, 1(8476):307-310.

19. Cordova CM, Schneider CR, Juttel ID, Cordova MM: Comparison of LDLcholesterol direct measurement with the estimate using the Friedewald formula in a sample of 10,664 patients. Arq Bras Cardiol 2004, 83:482-7.

20. Ran Jun K, Park HI, Chun S, Park H, Min WK: Effects of total cholesterol and triglyceride on the percentage difference between the lowdensity lipoprotein cholesterol concentration measured directly and calculated using the Friedewald formula. Clin Chem Lab Med 2008 46:371-5.

doi: 10.1186/1476-511X-9-52

Cite this article as: Chen et al., A modified formula for calculating low-density lipoprotein cholesterol values Lipids in Health and Disease 2010, 9:52

\section{Submit your next manuscript to BioMed Central} and take full advantage of:

- Convenient online submission

- Thorough peer review

- No space constraints or color figure charges

- Immediate publication on acceptance

- Inclusion in PubMed, CAS, Scopus and Google Scholar

- Research which is freely available for redistribution

Submit your manuscript at www.biomedcentral.com/submit
C) Biomed Central 